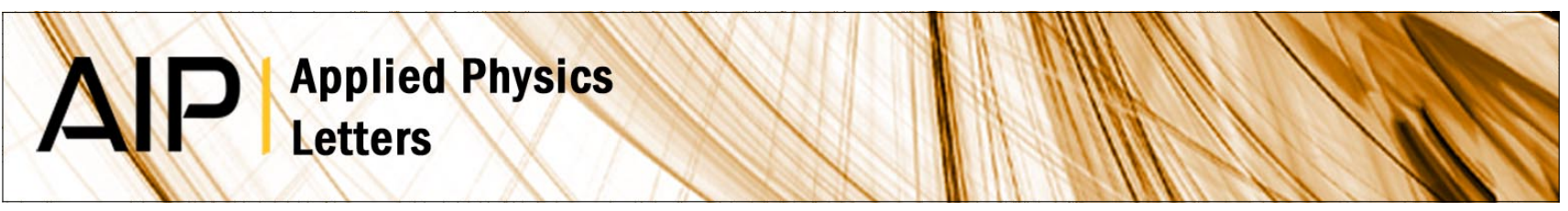

\title{
Efficient photonic crystal cavity-waveguide couplers
}

Andrei Faraon, Edo Waks, Dirk Englund, Ilya Fushman, and Jelena Vučković

Citation: Appl. Phys. Lett. 90, 073102 (2007); doi: 10.1063/1.2472534

View online: http://dx.doi.org/10.1063/1.2472534

View Table of Contents: http://apl.aip.org/resource/1/APPLAB/v90/i7

Published by the American Institute of Physics.

\section{Related Articles}

Terahertz mirage: Deflecting terahertz beams in an inhomogeneous artificial dielectric based on a parallel-plate waveguide

Appl. Phys. Lett. 101, 111108 (2012)

Integrating a plasmonic coupler to photo detector of terahertz frequency

Appl. Phys. Lett. 101, 091114 (2012)

Electromagnetically induced transparencies in a closed waveguide with high efficiency and wide frequency band Appl. Phys. Lett. 101, 093502 (2012)

Short-wavelength tunable Bragg reflectors based on coupling of propagating and cutoff waves: Modeling and experimental studies

Appl. Phys. Lett. 101, 083507 (2012)

Resonant transmission of light through $\mathrm{ZnO}$ nanowaveguides in a silver film

Appl. Phys. Lett. 101, 081113 (2012)

\section{Additional information on Appl. Phys. Lett.}

Journal Homepage: http://apl.aip.org/

Journal Information: http://apl.aip.org/about/about_the_journal

Top downloads: http://apl.aip.org/features/most_downloaded

Information for Authors: http://apl.aip.org/authors

\section{ADVERTISEMENT}

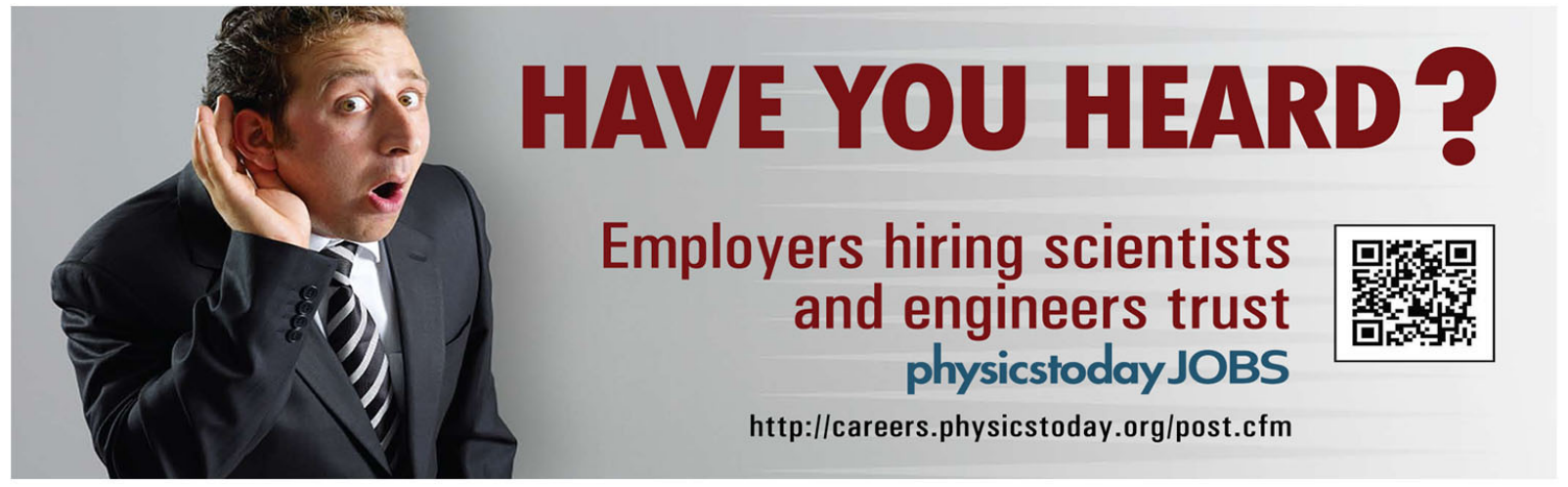




\title{
Efficient photonic crystal cavity-waveguide couplers
}

\author{
Andrei Faraon, ${ }^{\text {a) }}$ Edo Waks, ${ }^{\text {b) }}$ Dirk Englund, Ilya Fushman, and Jelena Vučković \\ E. L. Ginzton Laboratory, Stanford University, Stanford, California 94305
}

(Received 13 October 2006; accepted 11 January 2007; published online 12 February 2007)

\begin{abstract}
Coupling of photonic crystal (PC) linear three-hole defect cavities to PC waveguides is theoretically and experimentally investigated. The systems are designed to increase the overlap between the evanescent cavity field and the waveguide mode and to operate in the linear dispersion region of the waveguide. The simulations indicate increased coupling when the cavity is tilted by $60^{\circ}$ with respect to the waveguide axis, which was also confirmed by experiments. Up to $90 \%$ coupling efficiency into the waveguide was obtained. (C) 2007 American Institute of Physics. [DOI: 10.1063/1.2472534]
\end{abstract}

Structures that consist of InGaAs/GaAs quantum dots (QDs) coupled to two-dimensional photonic crystal (PC) cavities are promising candidates for highly efficient single photon sources. They represent essential devices for quantum cryptography and quantum computation. ${ }^{1-4}$ Efficient implementation of quantum computation devices requires on-chip integration of photonic circuits consisting of PC cavities and waveguides. ${ }^{5}$ The performance of these circuits is limited by the cavity-waveguide coupling efficiency. Our work investigates this coupling with the goal of improving the efficiency of single photon transfer into PC waveguides. The results are also relevant for channel drop filter applications in optical telecommunications.

We investigate the waveguide coupling efficiency of the linear three-hole defect cavities (L3). ${ }^{6}$ The L3 cavities have a high quality factor $(Q)$ to mode volume $(V)$ ratio and provide good matching between cavity and waveguide field patterns, which improves in-plane coupling efficiency. ${ }^{7,8}$ The cavity mode we work with has magnetic field with even/odd symmetry with respect to the $x / y$ axes. This mode [Fig. 1(a)] needs to be coupled to one of the guided modes in the PC waveguide. Of the possible waveguide bands inside the PC band gap, ${ }^{7}$ the best choice for coupling the L3 cavity is the one with similar symmetry and frequency as the L3 cavity mode [Fig. 1(b)]. For efficient coupling, the cavity and waveguide modes need to be spatially overlapped and frequency matched. A closer look at the L3 cavity field profile [Fig. 1(a)] reveals that the evanescent field is strongest along a direction tilted with respect to the cavity axis and is weak along the cavity axis. A good approach for obtaining a larger overlap between the cavity and waveguide mode is to tilt the cavity with respect to the waveguide axis by an angle of $60^{\circ}$ [Fig. 1(c)]. The choice of this angle is determined by the symmetry constraints of the triangular lattice. Directional couplers with cavity axes nonparallel to waveguide axes have recently been studied by Kim et al. ${ }^{9}$ and by Shinya et $a l{ }^{10,11}$ In contrast with previous work, we present here optimized designs of couplers as well as detailed theoretical and experimental data, confirming the advantage of the tilted configuration for coupling L3 cavities to PC waveguides.

To test the validity of our approach, we compare the coupling parameters for the tilted cavity configuration [Fig. 1(c)] to the standard approach where the cavity and the

\footnotetext{
${ }^{a)}$ Electronic mail: faraon@ stanford.edu

${ }^{b)}$ Present address: Department of Electrical and Computer Engineering, University of Maryland, College Park, MD 20742.
}

waveguide share the same axis (straight cavity configuration) [Fig. 1(d)]. First, three-dimensional finite difference time domain (3D FDTD) simulations of coupled cavity-waveguide systems were performed with both tilted and straight couplers. The frequency of the waveguide band was lowered with respect to the cavity frequency by reducing the size of the PC holes that bound the waveguide. In this way, coupling occurs in the dispersion-free linear region of the waveguide band. We directly simulated tilted and straight coupler configurations with spacing of two to five lattice hole separation between the cavity and the waveguide. The inset of Fig. 2 shows an image of the simulated magnetic field profile for a tilted cavity coupled to a waveguide with three-hole separation. In the tilted configuration, the separation between the cavity and the waveguide is changed along a direction indicated by the arrow in Fig. 1(c).

The energy transfer into the waveguide degrades the $Q$ of the coupled cavity. The total $Q$ of a coupled cavity relates to the uncoupled cavity quality factor $\left(Q_{c}\right)$ according to

$$
Q^{-1}=Q_{c}^{-1}+Q_{\mathrm{wg}}^{-1},
$$

where $Q_{\mathrm{wg}}^{-1}$ is the loss rate into the waveguide.

The coupling strength between the cavity and the waveguide is given by $1 / Q_{\mathrm{wg}}$ which is proportional to the decay rate of the cavity field into the waveguide. The quality factor
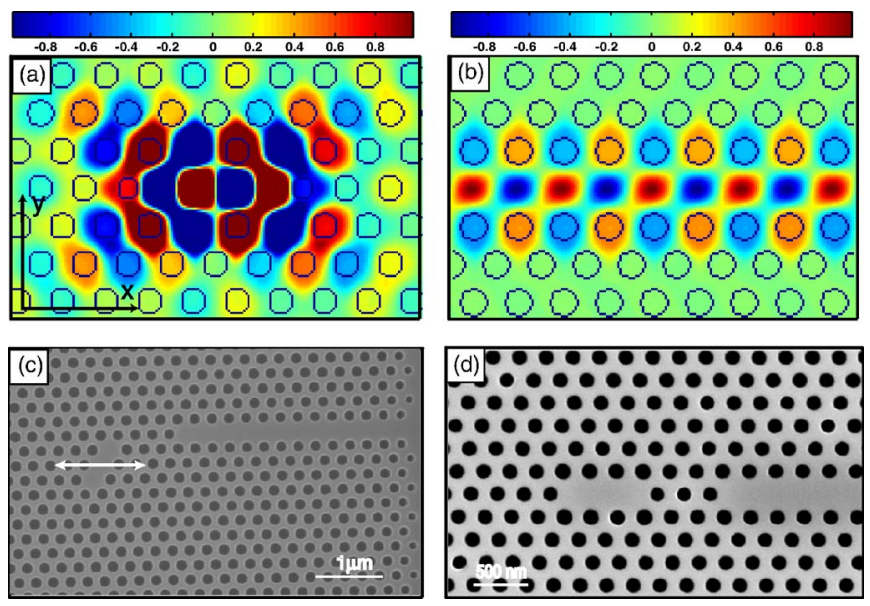

FIG. 1. (Color online) (a) Magnetic field ( $B_{z}$ component) for the mode with the highest quality factor in a L3 cavity. (b) Magnetic field pattern of the even mode in a PC waveguide. (c) Fabricated tilted cavity coupled to a waveguide (four-hole separation). In this experiment we shift the cavity with respect to the waveguide along the direction indicated by the arrow. (d) Fabricated straight cavity coupled to a waveguide (three-hole separation). 


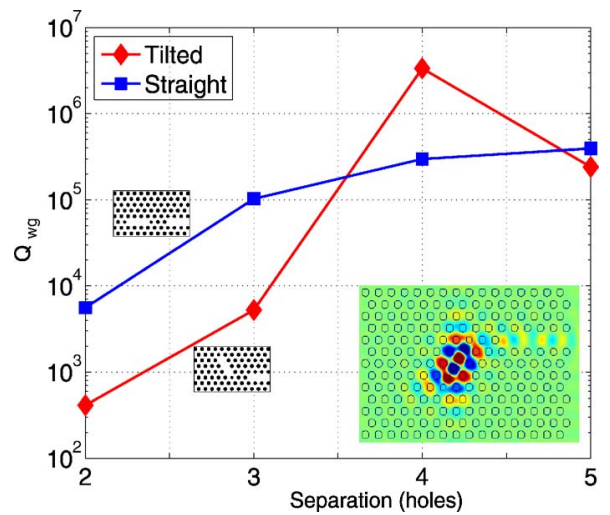

FIG. 2. (Color online) Simulation results for the cavity-waveguide coupling expressed in terms of the quality factor. The coupling strength is proportional to $\left(1 / Q_{\mathrm{wg}}\right)$. Simulated magnetic field of a cavity-waveguide coupler in tilted configuration with three-hole separation (inset).

$Q_{\mathrm{wg}}$ was computed from the 3D FDTD simulations, with results presented in Fig. 2. For the same cavity-waveguide separation, $Q_{\mathrm{wg}}$ is generally smaller for the tilted than for the straight configuration. This is an indication of better cavitywaveguide coupling obtained by tilting the cavity. One peculiar aspect of the simulations is that for the tilted coupling configuration, $Q$ is actually larger for four-hole than for fivehole separation. This is unexpected because it is natural to assume that reducing the distance between the cavity and waveguide would improve the overlap integral between the two modes. However, this increase in the quality factor is observed under a large variety of different simulation parameters, suggesting that it is real, as opposed to a simulation artifact. We suspect that, at four-hole separation, the antinode of one of the modes overlaps with the node of the other resulting in a lower overlap integral. Further investigation is required in order to conclusively confirm this.

The coupling changes from $Q_{\mathrm{wg}} \approx 500$ for the tilted cavity with two-hole separation to $Q_{\mathrm{wg}} \approx 10^{6}$ for four- and fivehole separations (both configurations). For single photon sources based on PC cavities with InGaAs QDs operating at $900-1000 \mathrm{~nm}$, the experimental out-of-plane quality factor is limited to about $Q_{c}=10^{4}$ because of material loss and fabrication imperfections. ${ }^{12}$ On the other hand, to get efficient photon transfer into the waveguide, $Q_{\mathrm{wg}}$ needs to be lower than $Q_{c}$; therefore, only the coupling configurations with two- and three-hole separations represent good options. Experimentally we expect the total $Q$ to be independent of the waveguide coupling in the case of four- and five-hole separations.

To test the validity of our simulation results, the couplers were fabricated on a $165 \mathrm{~nm}$ thick freestanding GaAs membrane containing an InGaAs QD layer. Structures with twoto five-hole separation in both tilted and straight configurations [Figs. 1(c) and 1(d)] were fabricated. We made seven structures of each kind. The spectrum of each cavity was measured using the InGaAs QDs embedded in the GaAs membrane as an internal light source. The fabrication and measurement procedures are similar with those reported in Ref. 3. The mean value of the quality factor for each configuration is plotted in Fig. 3(a), where the error bars are given by the standard deviation in $Q$ due to fabrication fluctuations between the seven structures of each kind.

As expected from simulations, the experimental data show that for the same cavity-waveguide separation, the total
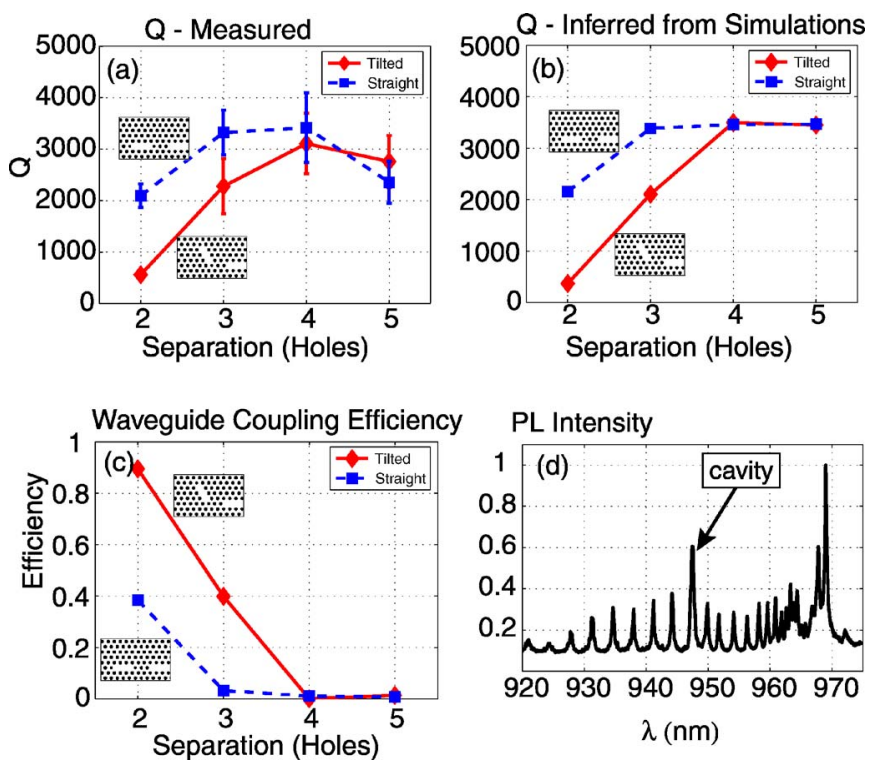

FIG. 3. (Color online) Comparison between simulations and experimental data for cavity-waveguide couplers. (a) Measured value of total cavity $Q$ (mean). (b) Value of total $Q$ inferred from simulations by combining simulated $Q_{\mathrm{wg}}$ and measured $Q_{c}$. (c) Coupling efficiency from the PC cavity into the PC waveguide. (d) Measured spectrum of a closed waveguide coupled to a L3 cavity. The Fabry-Pérot fringes are equidistant in the linear region of the waveguide dispersion relation (where the cavity is also located) and they get closer next to the waveguide band edge $(970 \mathrm{~nm})$.

quality factor is lower for the tilted than for the straight configuration. This result is a consequence of higher coupling for tilted cavities.

Since a more efficient coupling between the cavity and the waveguide degrades the cavity quality factor, when designing a PC network one should choose the configuration that gives the optimum trade-off between transfer efficiency and high $Q$. One advantage of using the tilted cavity is that the same set of parameters can be obtained with the cavity further spaced from the waveguide.

As mentioned before, the cavity coupling was designed to couple in the linear region of the waveguide-band dispersion relation. To test the position of the cavity with respect to the waveguide band, we fabricated longer waveguides closed at the ends. These waveguides act as Fabry-Pérot resonators. Fringes can be observed using the broad distribution of the QDs. ${ }^{13}$ In the linear region of the dispersion relation the fringes are equally spaced and get closer together as the frequency approaches the band edge. Since the cavity resonance was positioned in the region with equidistant fringes, we concluded that the coupling occurs in the linear region [Fig. $3(\mathrm{~d})]$.

For a direct comparison between simulation and experiment, $Q_{c}$ of the uncoupled cavity needs to be known. The upper bound for $Q_{c}$ is limited by fabrication imperfections and material loss. Our simulation results indicate that in the case of coupled cavities with four-hole separation, the coupling into the waveguide is very small so the total $Q$ is well approximated by $Q_{c}$. For this reason, the average value of the measured $Q$ for the tilted configuration with four-hole separation, was used as $Q_{c}$. By plugging $Q_{c}$ and the simulated value for $Q_{\mathrm{wg}}$ into expression (1), the predicted value for the total $Q\left(Q_{\text {tot }}\right)$ was computed. The values for $Q$ inferred from simulations are plotted in Fig. 3(b) and show good agreement with the experimental data [Fig. 3(a)]. Some inconsis- 
tency is observed in the case of five-hole separation. These inconsistencies result from fabrication errors.

The coupling efficiency into the waveguide was computed by taking the ratio $Q / Q_{\mathrm{wg}}$ and the results are plotted in Fig. 3(c). The coupling efficiency is up to $\eta \approx 40 \%$ in the case of straight configuration with two-hole separation and up to $\eta \approx 90 \%$ for tilted configuration with two-hole separation. Because of the coupling to the waveguide, the total cavity $Q$ dropped to $Q \approx 500$ for the $90 \%$ coupling efficiency case. However, the small mode volume of the L3 cavity still enables large Purcell factors $(F \approx 50)$. With $\eta \approx 90 \%$ and $F$ $\approx 50$ an efficient waveguide coupled single photon source with repetition rate of tens of gigahertz can be built. As discussed in Ref. 14, these are two parameters that in addition to multiphoton probability suppression (which is unaffected by the cavity) determine the quality of a single photon source for most applications; a higher $Q$ cavity will not improve these properties, as the repetition rate will still be limited to tens of gigahertz by the carrier capture time.

In conclusion we have designed PC cavity-waveguide couplers with optimized coupling efficiency and operating in the linear waveguide dispersion region. We have shown both theoretically and experimentally that the coupling between a L3 PC cavity and PC waveguides can be improved by tilting the cavity with respect to the waveguide. The coupling is more efficient because the evanescent tails of the cavity field are not oriented along the cavity axis but at a $30^{\circ}$ angle. Understanding and controlling the coupling mechanism are essential for on-chip single photon transfer and the implementation of on-chip quantum networks.

Financial support was provided by the MURI Center for Photonic Quantum Information Systems (ARO/DTO Program No. DAAD19-03-1-0199), ONR Young Investigator Award, and NSF Grant No. CCF-0507295.

${ }^{1}$ J. Vučković, D. Fattal, C. Santori, G. S. Solomon, and Y. Yamamoto, Appl. Phys. Lett. 82, 2374 (2003).

${ }^{2}$ E. Waks and J. Vučković, Phys. Rev. Lett. 96, 153601 (2006).

${ }^{3}$ D. Englund, D. Fattal, E. Waks, G. Solomon, B. Zhang, T. Nakaoka, Y. Arakawa, Y. Yamamoto, and J. Vučković, Phys. Rev. Lett. 95, 013904 (2005).

${ }^{4}$ B. Sanders, J. Vučković, and P. Grangier, Europhys. News 36, 56 (2005).

${ }^{5}$ D. Englund, A. Faraon, B. Zhang, Y. Yamamoto, and J. Vučković, e-print arXiv/ quant-ph/0609053.

${ }^{6}$ Y. Akahane, T. Asano, B.-S. Song, and S. Noda, Nature (London) 425, 944 (2003)

${ }^{7}$ D. Englund, I. Fushman, and J. Vučković, Opt. Express 12, 5961 (2005).

${ }^{8}$ E. Waks and J. Vučković, Opt. Express 13, 5064 (2005).

${ }^{9}$ G. H. Kim, Y. H. Lee, A. Shinya, and M. Notomi, Opt. Express 12, 6624 (2004).

${ }^{10}$ A. Shinya, S. Mitsugi, T. Tanabe, M. Notomi, I. Yokohama, H. Takara, and S. Kawanishi, Opt. Express 14, 1230 (2006).

${ }^{11}$ A. Shinya, S. Mitsugi, E. Kuramochi, and M. Notomi, Opt. Express 13 (2005).

${ }^{12}$ D. Englund and J. Vučković, Opt. Express 14, 3472 (2006).

${ }^{13}$ X. Letartre, C. Seassal, C. Grillet, P. Rojo-Romero, P. Viktorovitch, M. Le Vassor d'Yerville, D. Cassagne, and C. Jouanin, Appl. Phys. Lett. 79, 2312 (2001).

${ }^{14}$ J. Vučković, D. Englund, D. Fattal, E. Waks, and Y. Yamamoto, Physica E (Amsterdam) 31 (2006). 